\title{
Chiroptical Properties of Organic Radical Cations. The Electronic and Vibrational Circular Dichroism Spectra of $\alpha$-Tocopherol Derivatives and Sterically Hindered Chiral Hydroquinone Ethers
}

\author{
Tadashi Mori $*,+$ Hiroshi Izumi, $\neq$ and Yoshihisa Inoue*,,$+\mathbb{\Phi}$
}

\section{Experimental Section}

General. All starting materials, reagents, and solvents were commercially available and used without further purification, unless otherwise stated. Chiral $(S)-(+)$-2-butanol (99\%) was used as obtained from Aldrich. Dichloromethane (Nacalai) was repeatedly stirred with fresh aliquots of concentrated sulfuric acid $(\sim 10 \% \mathrm{v} / \mathrm{v})$, until the acid layer remained clear. After separation, it was washed with water, aqueous sodium bicarbonate, brine, and water, and then dried over calcium chloride. The dried dichloromethane was distilled twice from $\mathrm{P}_{2} \mathrm{O}_{5}$ under argon atmosphere and stored in a Schlenk tube equipped with a Teflon valve fitted with Viton $O$-rings. Nitrosonium tetrafluoroborate (Aldrich) was recrystallized from acetonitrile. Tetrahydrofuran (THF, Wako) was distilled from sodium/benzophenone. Anhydrous toluene (Wako) and hexane (Wako) were distilled from calcium hydride under Ar and stored in Schlenk flasks. Column chromatography was performed over 70-230 mesh silica gel from Merck or by a GPC column (Jaigel $1-\mathrm{H}$ and 2-H from Japan Analytical Industry Co.) with chloroform as an eluent. ${ }^{1} \mathrm{H}-\mathrm{NMR}(400 \mathrm{MHz})$ and ${ }^{13} \mathrm{C}-\mathrm{NMR}(100 \mathrm{MHz})$ spectra were obtained on a JEOL EX400 or GSX-400 instrument. All chemical shifts are reported in ppm relative to $\mathrm{Me}_{4} \mathrm{Si}$ for ${ }^{1} \mathrm{H}(0 \mathrm{ppm})$ or solvent $\mathrm{CDCl}_{3}$ for ${ }^{13} \mathrm{C}(77.16 \mathrm{ppm})$ used as references, and the coupling constants $(J)$ in Hz. IR spectra were obtained on a JASCO FT/IR-230 spectrometer and UV-vis spectra on JASCO V-550 equipped with a temperature controller. Gas chromatographic analyses were performed on a Shimadzu GC-14B instrument fitted with a C-R6A integrator using a capillary column (SUPELCO, SPB5, $30 \mathrm{~m} \times 0.25 \mathrm{~mm}$ i.d.). Melting points were measured with a Yanaco MP-21 apparatus and are uncorrected. Mass spectra were obtained on a JEOL JMS-DX-303 instrument. Elemental analysis was performed at the Center for Chemical Analyses, Osaka University. Circular dichroism (CD) spectra were measured in a conventional quartz cell (light path $1 \mathrm{~cm}$ ) on a J-720WI spectropolarimeter equipped with a PTC-348WI temperature controller. Aqueous solution of $(+)$-ammonium camphorsulfonate- $d_{10}$ $(0.06 \%)$ was used for a calibration of the spectrometer sensitivity $(\theta=190.4 \mathrm{mdeg}$ at 
$290.5 \mathrm{~nm}$ ). $\Delta$-Tris(ethylenediamine) cobalt(III) was further employed for a calibration of the visible region $(\Delta \varepsilon=+1.89$ at $490 \mathrm{~nm}) .{ }^{1}$ ECD spectra were measured in a quartz cuvette with a Teflon screw cock. VCD (and IR) spectra of $\mathbf{2 b}$ and $\mathbf{2} \mathbf{b}^{\mathbf{}}{ }^{+}(0.22 \mathrm{M})$ were obtained in dichloromethane- $d_{2}$ at ambient temperature on a Chiralir (Bomem-BioTools) fitted with $\mathrm{ZnSe}$ beam splitter, $\mathrm{BaF}_{2}$ polarizer, optical filter, and $2 \times$ $2 \mathrm{~mm} \mathrm{HgCdTe}$ detector. The sample solution was placed in $\mathrm{BaF}_{2}$ cell $(2 \mathrm{~cm} \phi$ and 72 $\mu \mathrm{m}$ light path). The VCD/IR signals were accumulated for $1-6 \mathrm{~h}$ at a $4 \mathrm{~cm}^{-1}$ resolution, and the data obtained in first $3 \mathrm{~h}$ were averaged in a typical run.

The theoretical IR absorption and VCD spectra were simulated with Lorentzian band shapes of $6 \mathrm{~cm}^{-1}$ full width at half-mean, and the frequencies were scaled by a factor of 0.97 or 0.91 for B3LYP/6-31G(d) or HF/3-21G* calculations, respectively. The optical rotations were measured with a JASCO DIP-1000 Digital Polarimeter with a thermostated $10 \mathrm{~cm}$ cell at the sodium D-line $(589.3 \mathrm{~nm})$. Cyclic voltammetry was performed on a BAS CV-100B electrochemical analyzer. Hydroquinone donor $\mathbf{1 b}$ (1 $\mathrm{mM})$ and tetra- $n$-butylammonium hexafluorophosphate $(0.2 \mathrm{M})$ as electrolyte were placed in a gas-tight Schlenk-type separated cell under Ar. Anodic and cathodic peak potentials were measured with a Pt electrode in anhydrous dichloromethane at a sweep rate of $0.2 \mathrm{~V} \mathrm{~s}^{-1}$. The $\mathrm{Ag}^{+} / \mathrm{AgNO}_{3}$ reference electrode was used and calibrated with ferrocene $\left(E_{1 / 2}=0.02 \mathrm{~V}\right)$, and the measured potential was converted to the voltage against the SCE by means of the relationship: $E(\mathrm{SCE})=E\left(\mathrm{Ag}^{+} / \mathrm{AgNO}_{3}\right)+0.25 \mathrm{~V}$. All the calculations were performed using the GAUSSIAN 98 software package ${ }^{2}$ running on 16-CPU parallel Pentium IV processors at the Handai FRC Computer Center. The calculations were performed using either the Hartree-Fock method with B3LYP/6-311+G(2d,p)//HF/3-21G* or DFT method with B3LYP/6-311+G(2d,p)//B3LYP/6-31G(d). The calculated energies were corrected for

(1) McCaffery, A. J.; Mason, S. F. Mol. Phys. 1963, 6, 359.

(2) Frisch, M. J.; Trucks, G. W.; Schlegel, H. B.; Scuseria, G. E.; Robb, M. A.; Cheeseman, J. R.; Zakrzewski, V. G.; Montgomery, J. A., Jr.; Stratmann, R. E.; Burant, J. C.; Dapprich, S.; Millam, J. M.; Daniels, A. D.; Kudin, K. N.; Strain, M. C.; Farkas, O.; Tomasi, J.; Barone, V.; Cossi, M.; Cammi, R.; Mennucci, B.; Pomelli, C.; Adamo, C.; Clifford, S.; Ochterski, J.; Petersson, G. A.; Ayala, P. Y.; Cui, Q.; Morokuma, K.; Malick, D. K.; Rabuck, A. D.; Raghavachari, K.; Foresman, J. B.; Cioslowski, J.; Ortiz, J. V.; Baboul, A. G.; Stefanov, B. B.; Liu, G.; Liashenko, A.; Piskorz, P.; Komaromi, I.; Gomperts, R.; Martin, R. L.; Fox, D. J.; Keith, T.; Al-Laham, M. A.; Peng, C. Y.; Nanayakkara, A.; Gonzalez, C.; Challacombe, M.; Gill, P. M. W.; Johnson, B.; Chen, W.; Wong, M. W.; Andres, J. L.; Gonzalez, C.; Head-Gordon, M.; Replogle, E. S.; Pople, J. A.; Gaussian 98, Revision A.11; Gaussian, Inc.: Pittsburgh, PA, 1998. 
the zero point energies obtained by frequency calculations, which were empirically scaled by a factor of 0.98 or 0.94 for B3LYP/6-31G(d) or HF/3-21G* calculations, respectively.

Synthesis of $\left(2 R, 4^{\prime} R, 8^{\prime} R\right)$-methyl- $\alpha$-tocopherol $1 \mathrm{~b}$. Optically active methylated $\alpha$-tocopherol (MeVE) was synthesized by direct methylation of $(+)-\alpha$-tocopherol as reported previously for the optically inactive analogue. ${ }^{3}(+)$ - $\alpha$-Tocopherol (Sigma, Lot. No. 79H1390, $10.0 \mathrm{~g}, 16 \mathrm{mmol})$ was dissolved in THF $(120 \mathrm{~mL})$, to which $\mathrm{NaH}$ in oil $(\sim 60 \%, 0.77 \mathrm{~g}, 20 \mathrm{mmol})$ was added in one portion at an ambient temperature under an argon atmosphere. The solution was stirred for $1 \mathrm{~h}$ and iodomethane (1.6 mL, $24 \mathrm{mmol})$ was added and refluxed for $12 \mathrm{~h}$. The resulting solution was poured into aqueous ammonium chloride and the mixture was extracted with ether $(2 \times 100 \mathrm{~mL})$. The ether extract was washed with water and brine, and then dried over sodium sulfate. After filtration and evaporation, the crude material obtained was chromatographed on silica gel with hexane-ethyl acetate (50:1) as an eluent, affording an oily material, this was further purified with an alumina column to afford colorless pure sample in $50 \%(5.2 \mathrm{~g})$.

(2R,4'R,8' $\boldsymbol{R})$-methyl- $\alpha$-tocopherol (1b): colorless oil; ${ }^{1} \mathrm{H}$ NMR $\delta 0.87$ (3H, d, $J=$ 6.5), $0.88(3 \mathrm{H}, \mathrm{d}, J=6.5), 0.89(6 \mathrm{H}, \mathrm{d}, J=6.5), 1.02-1.64(21 \mathrm{H}, \mathrm{m}), 1.26(3 \mathrm{H}, \mathrm{s}), 1.81$ $(2 \mathrm{H}$, sym. m, $J=6.9), 2.11(3 \mathrm{H}, \mathrm{s}), 2.16(3 \mathrm{H}, \mathrm{s}), 3.65(3 \mathrm{H}, \mathrm{s}) ;{ }^{13} \mathrm{C}$ NMR $\delta 11.82,11.90$, $12.69,19.82$, 19.90, 20.79, 21.18, 22.78, 22.87, 24.04, 24.60, 24.96, 28.13, 31.44, 32.86, $32.96,37.44,37.57,37.61,37.63,39.53,40.21,60.52$, 74.91, 117.66, 123.03, 125.83, 127.86, 147.91 149.52; Anal. Calcd for $\mathrm{C}_{30} \mathrm{H}_{52} \mathrm{O}_{2}$ : C, 81.02; H, 11.79. Found: C, 80.96; H, 11.76; EI-MS (70 eV) $m / z 445\left(\mathrm{M}^{+}+1,39 \%\right), 444\left(\mathrm{M}^{+}, 100\right), 179(36) \cdot[\alpha]_{\mathrm{D}}{ }^{30}(c$ $\left.1.01, \mathrm{CHCl}_{3}\right)+0.55^{\circ}$.

\section{Synthesis}

of

\section{9,10-Bis((R)-1-methylpropyloxy)-1,4:5,8-dimethano-1,2,3,4,5,6,7,8-octahydroanthr}

ancene 2b. This compound was prepared by the Mitsunobu reaction of the corresponding hydroquinone derivative

(9,10-dihydroxy-1,4:5,8-dimethano-1,2,3,4,5,6,7,8-octahydroanthrancene) ${ }^{4,5}$ with a slight excess of chiral alcohol. Hydroquinone (1.32 g, $5.4 \mathrm{mmol}),(S)-(+)$-2-butanol (3.0 $\mathrm{mL}, 32 \mathrm{mmol})$ and triphenylphosphine $(8.63 \mathrm{~g}, 51 \mathrm{mmol})$ were dissolved in THF (100 $\mathrm{mL}$ ) under Ar at room temperature, and diisopropyl azodicarboxylate $(4.3 \mathrm{~mL}, 22$ mmol) was added dropwise over $1 \mathrm{~h}$. The solution was stirred for $5 \mathrm{~d}$ and the solvent

(3) Mori, T.; Takamoto, M.; Wada, T.; Inoue, Y. Helv. Chim. Acta 2001, 84, 2693.

(4) Rathore, R.; Kochi, J. K. J. Org. Chem. 1995, 60, 4399.

(5) Muffett, R. B. Org. Synth., Coll. Vol. IV 1963, 238. 
was removed in vacuo. The residue obtained was chromatographed on silica gel with dichloromethane as an eluent. Further purification was performed with recycling GPC. The desired hydroquinone ether was obtained as the first fraction in $11 \%$ yield $(0.20 \mathrm{~g})$, while the second fraction (obtained in $37 \%$ ) was an oxidation product. $2 \mathbf{b}: \mathrm{mp}$ $71-72{ }^{\circ} \mathrm{C} ;{ }^{1} \mathrm{H}$ NMR $\delta 0.95(3 \mathrm{H}, \mathrm{t}, J=7.6), 1.08(3 \mathrm{H}, \mathrm{t}, J=7.4), 1.16(3 \mathrm{H}, \mathrm{d}, J=6.2)$, $1.31(3 \mathrm{H}, \mathrm{d}, J=6.2), 1.40(2 \mathrm{H}, \mathrm{d}, J=8.3), 1.84(4 \mathrm{H}, \mathrm{d}, J=8.7), 1.00-1.85(10 \mathrm{H}, \mathrm{m})$, 3.47 (4H, br s), $3.94(1 \mathrm{H}$, sym m, $J=6.2), 4.01(1 \mathrm{H}$, sym $\mathrm{m}, J=6.2) ;{ }^{13} \mathrm{C} \mathrm{NMR} \delta 10.08$, 10.39 , 19.55, 20.27, 26.99, 27.00, 29.26, 30.26, 41.10, 49.22, 79.86, 79.96, 138.47, 138.89, 141.11, 141.42; IR $\left(\mathrm{CaF}_{2}, \mathrm{v} / \mathrm{cm}^{-1}\right): 2966,2868,1464,1374,1374,1309,1201$, 1107, 754, 708; Anal. Calcd for $\mathrm{C}_{24} \mathrm{H}_{34} \mathrm{O}_{2}$ : C, 81.31; H, 9.67. Found: C, 81.09; H, 9.52; EI-MS (70 eV) m/z 354 (M+, 49\%), 298 (19), 185 (15), 184 (100), 92 (67); HRMS: found 354.2556, expected 354.2559. $[\alpha]_{\mathrm{D}}^{28}\left(c 1.03, \mathrm{CHCl}_{3}\right)=-8.4^{\circ}$.

Preparation of radical cation with triethyloxonium hexachloroantimonate $\left[\mathbf{E t}_{3} \mathbf{O}^{+} \mathbf{S b C l}_{6}{ }^{-}\right]$. A typical procedure. A $100-\mathrm{mL}$ flaskequipped with a Schlenk adapter was charged with triethyloxonium hexachloroantimonate $(1.5 \mathrm{mmol})$, and a solution of the pure organic donor $(1 \mathrm{mmol})$ in freshly prepared anhydrous dichloromethane $(25$ $\mathrm{mL}$ ) was added under an argon atmosphere at $0{ }^{\circ} \mathrm{C}$. The heterogeneous mixture immediately took on an organge coloration which intensified with time. The dark colored mixture was stirred for $2 \mathrm{~h}$ to yield a solution of the radical cation. The solution was cooled to $-20{ }^{\circ} \mathrm{C}$ in an ice-salt bath, and anhydrous diethyl ether $(50 \mathrm{~mL})$ was added to precipitate the dissolved salt. The orange precipitate was filtered under an argon atmosphere, washed with diethyl ether $(3 \times 25 \mathrm{~mL})$, and dried in vacuo. The pure cation radical salt was obtained in essentially quantitative yield $(>95 \%)$ and was photospectrometrically identical to that obtained. Purity of the radical cation was examined by iodometry as described previously ${ }^{6}$ and found that greater than $98 \%$. The purity was also determined spectrophotometrically and yield was essentially quantitative.

Preparation of radical cations using $\mathrm{NO}_{+} \mathbf{B F}_{4}{ }^{-}$. A typical procedure. $50 \mathrm{~mL}$ flask fitted with a Schlenk adaptor was charged with nitrosonium tetrafluoroborate $(0.5$ $\mathrm{mmol})$, and a cold solution of donor $(0.5 \mathrm{mmol})$ in anhydrous dichloromethane $(20 \mathrm{~mL})$ was added under an argon atmosphere at $-10{ }^{\circ} \mathrm{C}$. The solution immediately took on an orange coloration, and the solution was stirred (while slowly bubbling argon through the

(6) Rathore, R.; Kumar, A. S.; Lindeman, S. V.; Kochi, J. K. J. Org. Chem. 1998, 63, 5847. 
solution to entrain gaseous NO) for $10 \mathrm{~min}$ to yield a solution of cation radical. The identity of gaseous nitric oxide (NO) produced above was confirmed by UV-vis spectral analysis of the gas, which revealed the characteristic absorbances of NO at $\lambda_{\max } 204$, 214, and $226 \mathrm{~nm}$. The spectrophotometric analysis of the red solution indicated the quantitative formation of corresponding radical cation salt. The solution was carefully layered with dry toluene $(30 \mathrm{~mL})$ and placed in a refrigerator $\left(-20{ }^{\circ} \mathrm{C}\right)$. During the course of 3 days, orange crystals of the cation-radical salt were deposited which were filtered under argon and dried in vacuo $(\sim 80 \%)$. The purity of the radical cation was analyzed as above. 
Table S1 Ab initio Calculations of Annulated Hydroquinone Ethers (2 and 3), and Their Radical Cations in anti/syn Conformations ${ }^{a}$

\begin{tabular}{|c|c|c|c|c|}
\hline \multirow[b]{2}{*}{ compound } & \multirow[b]{2}{*}{ anti } & \multicolumn{2}{|c|}{ HF energy / Hartrees } & \multirow{2}{*}{$\begin{array}{l}\text { rough } \\
\text { estimation of the } \\
\text { anti/syn ratio }\end{array}$} \\
\hline & & syn & $\overline{\Delta G / \mathrm{kcal} \mathrm{mol}} \mathrm{m}^{-1}$ & \\
\hline $2 \mathbf{a}$ & -849.4722710 & -849.4723830 & +0.07 & 0.89 \\
\hline $2 a^{\bullet+}$ & -849.2241177 & -849.2223189 & -1.13 & 6.7 \\
\hline $2 \mathbf{b}$ & -1085.274800 & -1085.272771 & -1.27 & 8.6 \\
\hline $2 \mathbf{b}^{\bullet+}$ & -1085.296753 & -1085.295491 & -0.79 & 3.8 \\
\hline $2 \mathbf{b}^{b}$ & -1085.035772 & -1085.033853 & -1.20 & 7.6 \\
\hline $\mathbf{2} \mathbf{b}^{\bullet+b}$ & -1085.057008 & -1085.054091 & -1.83 & 22 \\
\hline $2 \mathrm{c}$ & -1085.272774 & -1085.274574 & +1.13 & 0.15 \\
\hline $2 c^{\bullet+}$ & -1085.035780 & -1085.032263 & -2.21 & 42 \\
\hline $\mathbf{3 a}$ & -928.0929170 & -928.0931270 & +0.13 & 0.8 \\
\hline $3 \mathbf{a}^{\bullet+}$ & -927.8412320 & -927.8326597 & -5.38 & $>100$ \\
\hline $3 \mathbf{b}$ & -1163.885289 & -1163.893296 & +5.02 & $<0.01$ \\
\hline $3 \mathbf{b}^{\bullet+}$ & -1163.646407 & -1163.643117 & -2.06 & 33 \\
\hline $3 \mathbf{c}$ & -1163.892368 & -1163.893191 & +0.52 & 0.42 \\
\hline $3 c^{\bullet+}$ & -1163.648808 & -1163.645117 & -2.32 & 50 \\
\hline
\end{tabular}

${ }^{a}$ All ab initio calculations were performed at the B3LYP/6-311+G(2d,p)//HF/3-21G* level, unless otherwise indicated. ${ }^{b}$ At B3LYP/6-311+G(2d,p)//B3LYP/6-31G(d) level. 
Table S2 Ab initio Calculations of a Complete Set of Conformers of 9,10-Bis((R)-1-methylpropyloxy)-1,4:5,8-dimethano-1,2,3,4,5,6,7,8-octahydroanthrance ne $\mathbf{2 b}$ and Radical Cation $\mathbf{2} \mathbf{b}^{++}$

\begin{tabular}{llllll}
\hline conformation & $\begin{array}{l}\text { optimized HF } \\
\text { energy / Hartree }\end{array}$ & $\begin{array}{l}\text { zero point } \\
\text { energy (ZPE) }\end{array}$ & $\begin{array}{l}\mathrm{HF} \text { energy correlated } \\
\text { with ZPE / } / \mathrm{kcal} \mathrm{mol}^{-1}\end{array}$ & $\begin{array}{l}\Delta \mathrm{G} / \\
\mathrm{kcal} \mathrm{mol}\end{array}$ & population
\end{tabular}

2b at B3LYP/6-311G+(2d,p)//B3LYP/6-31G(d) level

$\begin{array}{llllll}\text { anti }\left(\mathrm{G}^{+}, \mathrm{G}^{+}\right) & -1085.76857642 & 0.481256 & -681034.022850248 & 0.468580169 & 11.96 \% \\ \text { anti }\left(\mathrm{G}^{+}, \mathrm{T}\right) & -1085.76975371 & 0.481948 & -681034.335885370 & 0.155545047 & 16.35 \% \\ \text { anti }\left(\mathrm{G}^{+}, \mathrm{G}^{-}\right) & -1085.76781559 & 0.482472 & -681032.797326453 & 1.694103963 & 3.51 \% \\ \text { anti }(\mathrm{T}, \mathrm{T}) & -1085.77010747 & 0.482056 & -681034.491430417 & 0.000000000 & 19.10 \% \\ \text { anti }\left(\mathrm{T}, \mathrm{G}^{-}\right) & -1085.76908947 & 0.481571 & -681034.151002748 & 0.340427669 & 13.59 \% \\ \text { anti }\left(\mathrm{G}^{-}, \mathrm{G}^{-}\right) & -1085.76759346 & 0.482768 & -681032.475835515 & 2.015594902 & 2.55 \% \\ \operatorname{syn}\left(\mathrm{G}^{+}, \mathrm{G}^{+}\right) & -1085.76656683 & 0.480493 & -681033.231218901 & 1.260211516 & 5.42 \% \\ \operatorname{syn}\left(\mathrm{G}^{+}, \mathrm{T}\right) & -1085.76950512 & 0.481956 & -681034.174971101 & 0.316459316 & 13.92 \% \\ \operatorname{syn}\left(\mathrm{G}^{+}, \mathrm{G}^{-}\right) & -1085.76829082 & 0.481381 & -681033.766732245 & 0.724698171 & 9.25 \% \\ \operatorname{syn}(\mathrm{T}, \mathrm{T}) & -1085.76702426 & 0.482916 & -681032.027605981 & 2.463824435 & 1.63 \% \\ \operatorname{syn}\left(\mathrm{T}, \mathrm{G}^{-}\right) & -1085.76395428 & 0.482019 & -681030.653008018 & 3.838422399 & 0.41 \% \\ \operatorname{syn}\left(\mathrm{G}^{-}, \mathrm{G}^{-}\right) & -1085.76693522 & 0.482251 & -681032.380847394 & 2.110583023 & 2.31 \%\end{array}$

2b at B3LYP/6-311G+(2d,p)//HF/3-21G* level

$\begin{array}{llllll}\text { anti }\left(\mathrm{G}^{+}, \mathrm{G}^{+}\right) & -1085.76436383 & 0.520314 & -681020.247353697 & 0.000000000 & 24.27 \% \\ \text { anti }\left(\mathrm{G}^{+}, \mathrm{T}\right) & -1085.76434748 & 0.521020 & -681019.820254793 & 0.427098904 & 15.84 \% \\ \text { anti }\left(\mathrm{G}^{+}, \mathrm{G}^{-}\right) & -1085.76308492 & 0.520772 & -681019.174411473 & 1.072942224 & 8.30 \% \\ \text { anti }(\mathrm{T}, \mathrm{T}) & -1085.76497422 & 0.521941 & -681019.669759880 & 0.577593818 & 13.62 \% \\ \text { anti }\left(\mathrm{T}, \mathrm{G}^{-}\right) & -1085.76395227 & 0.521486 & -681019.297119324 & 0.950234373 & 9.39 \% \\ \text { anti }\left(\mathrm{G}^{-}, \mathrm{G}^{-}\right) & -1085.76228691 & 0.521184 & -681018.430398057 & 1.816955640 & 3.95 \% \\ \operatorname{syn}\left(\mathrm{G}^{+}, \mathrm{G}^{+}\right) & -1085.76199402 & 0.519952 & -681018.974008784 & 1.273344913 & 6.79 \% \\ \operatorname{syn}\left(\mathrm{G}^{+}, \mathrm{T}\right) & -1085.76234747 & 0.521261 & -681018.422937409 & 1.824416289 & 3.92 \% \\ \operatorname{syn}\left(\mathrm{G}^{+}, \mathrm{G}^{-}\right) & -1085.76306561 & 0.520614 & -681019.255581207 & 0.991772490 & 9.00 \% \\ \operatorname{syn}(\mathrm{T}, \mathrm{T}) & -1085.76139912 & 0.521745 & -681017.542073709 & 2.705279988 & 1.62 \% \\ \operatorname{syn}\left(\mathrm{T}, \mathrm{G}^{-}\right) & -1085.76026087 & 0.521124 & -681017.194464131 & 3.052889566 & 1.15 \% \\ \operatorname{syn}\left(\mathrm{G}^{-}, \mathrm{G}^{-}\right) & -1085.76187935 & 0.521779 & -681017.823348191 & 2.424005506 & 2.15 \%\end{array}$




$\begin{array}{llllll}\mathbf{2 b}^{\mathbf{0}^{+}} \text {at B3LYP/6-311G+(2d,p)/B3LYP/6-31G(d) level } & & & \\ \text { anti }\left(\mathrm{G}^{+}, \mathrm{G}^{+}\right) & -1085.52955592 & 0.481995 & -680883.580575381 & 0.000000000 & 22.61 \% \\ \text { anti }\left(\mathrm{G}^{+}, \mathrm{T}\right) & -1085.52946064 & 0.482476 & -680883.224870115 & 0.355705266 & 15.84 \% \\ \text { anti }\left(\mathrm{G}^{+}, \mathrm{G}^{-}\right) & -1085.52860221 & 0.483160 & -680882.265393280 & 1.315182101 & 6.07 \% \\ \text { anti }(\mathrm{T}, \mathrm{T}) & -1085.53017516 & 0.482672 & -680883.552656982 & 0.027918400 & 21.99 \% \\ \text { anti }\left(\mathrm{T}, \mathrm{G}^{-}\right) & -1085.52936579 & 0.483032 & -680882.823293905 & 0.757281477 & 10.60 \% \\ \text { anti }\left(\mathrm{G}^{-}, \mathrm{G}^{-}\right) & -1085.52715729 & 0.482357 & -680881.852706136 & 1.727869245 & 4.02 \% \\ \operatorname{syn}\left(\mathrm{G}^{+}, \mathrm{G}^{+}\right) & -1085.52669944 & 0.482057 & -680881.749964005 & 1.830611376 & 3.62 \% \\ \operatorname{syn}\left(\mathrm{G}^{+}, \mathrm{T}\right) & -1085.52746907 & 0.481994 & -680882.271672391 & 1.308902990 & 6.11 \% \\ \operatorname{syn}\left(\mathrm{G}^{+}, \mathrm{G}^{-}\right) & -1085.52568010 & 0.482739 & -680880.690745038 & 2.889830344 & 1.26 \% \\ \operatorname{syn}(\mathrm{T}, \mathrm{T}) & -1085.52641177 & 0.481224 & -680882.081918539 & 1.498656842 & 5.05 \% \\ \operatorname{syn}\left(\mathrm{T}, \mathrm{G}^{-}\right) & -1085.52461552 & 0.480910 & -680881.147930638 & 2.432644743 & 1.99 \% \\ \operatorname{syn}\left(\mathrm{G}^{-}, \mathrm{G}^{-}\right) & -1085.52506343 & 0.482739 & -680880.303778754 & 3.276796627 & 0.85 \%\end{array}$

$\mathbf{2 b}^{\text {•+ }}$ at B3LYP/6-311G+(2d,p)//HF/3-21G* level

\begin{tabular}{llllll} 
anti $\left(\mathrm{G}^{+}, \mathrm{G}^{+}\right)$ & -1085.52512477 & 0.520090 & -680870.254825682 & 0.000000000 & $23.99 \%$ \\
anti $\left(\mathrm{G}^{+}, \mathrm{T}\right)$ & -1085.52464078 & 0.520359 & -680869.792293387 & 0.462532295 & $15.11 \%$ \\
anti $\left(\mathrm{G}^{+}, \mathrm{G}^{-}\right)$ & -1085.52387138 & 0.520518 & -680869.215610211 & 1.039215471 & $8.49 \%$ \\
anti $(\mathrm{T}, \mathrm{T})$ & -1085.52495077 & 0.520551 & -680869.873453709 & 0.381371974 & $16.38 \%$ \\
anti $\left(\mathrm{T}, \mathrm{G}^{-}\right)$ & -1085.52442945 & 0.520635 & -680869.496724867 & 0.758100816 & $11.24 \%$ \\
anti $\left(\mathrm{G}^{-}, \mathrm{G}^{-}\right)$ & -1085.52272708 & 0.520875 & -680868.286769834 & 1.968055849 & $3.35 \%$ \\
$\operatorname{syn}\left(\mathrm{G}^{+}, \mathrm{G}^{+}\right)$ & -1085.52171513 & 0.518506 & -680869.050475313 & 1.204350369 & $7.19 \%$ \\
$\operatorname{syn}\left(\mathrm{G}^{+}, \mathrm{T}\right)$ & -1085.52235623 & 0.518810 & -680869.273282852 & 0.981542830 & $8.99 \%$ \\
$\operatorname{syn}\left(\mathrm{G}^{+}, \mathrm{G}^{-}\right)$ & -1085.52035260 & 0.518925 & -680867.948087269 & 2.306738414 & $2.39 \%$ \\
$\operatorname{syn}(\mathrm{T}, \mathrm{T})$ & -1085.51954165 & 0.518743 & -680867.546665551 & 2.708160131 & $1.60 \%$ \\
$\operatorname{syn}\left(\mathrm{T}, \mathrm{G}^{-}\right)$ & -1085.51788605 & 0.519097 & -680866.298750837 & 3.956074845 & $0.46 \%$ \\
$\operatorname{syn}\left(\mathrm{G}^{-}, \mathrm{G}^{-}\right)$ & -1085.51896780 & 0.519294 & -680866.861245772 & 3.393579910 & $0.81 \%$ \\
\hline
\end{tabular}




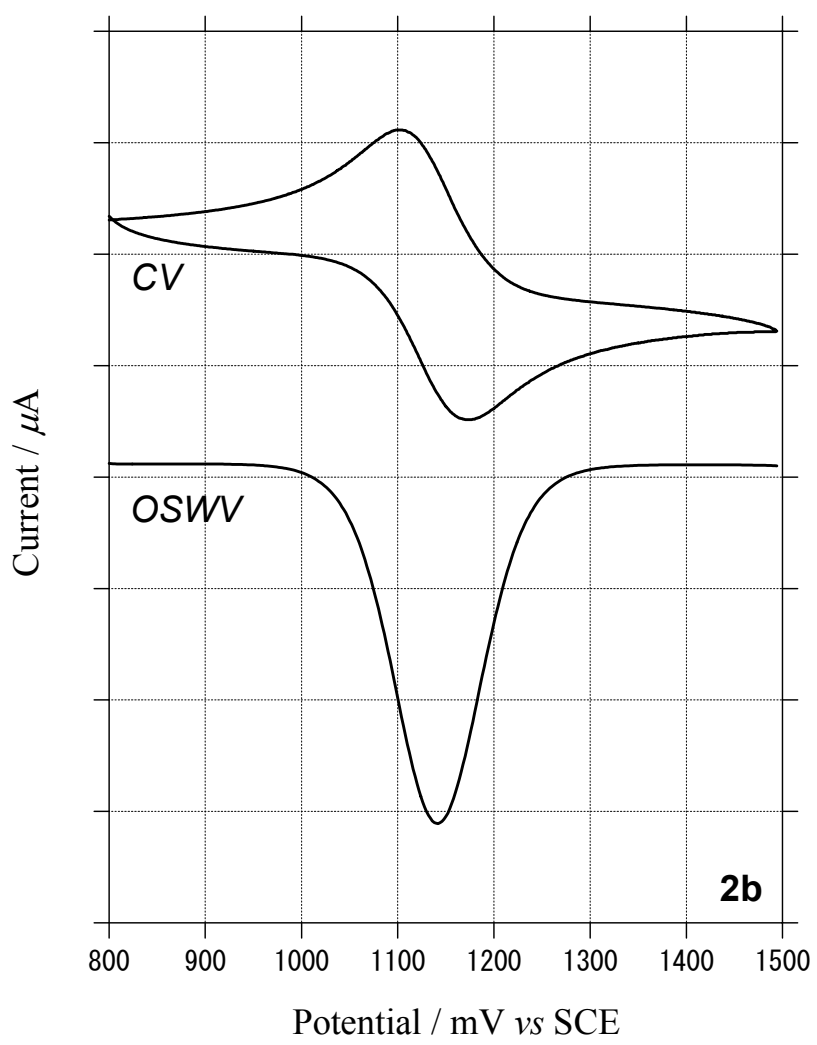

Figure S1 Cyclic voltammograms (top, scanning rate $=0.2 \mathrm{~V} \mathrm{~s}^{-1}$, Pt electrode) and Osteryoung square-wave voltammograms (bottom) taken with a $1 \mathrm{mM}$ dichloromethane solution of chiral hydroquinone ether $\mathbf{2 b}$ in dichloromethane containing $0.2 \mathrm{M}$ tetra- $n$-butylammonium hexafluorophosphate as an electrolyte at $25^{\circ} \mathrm{C}$. 


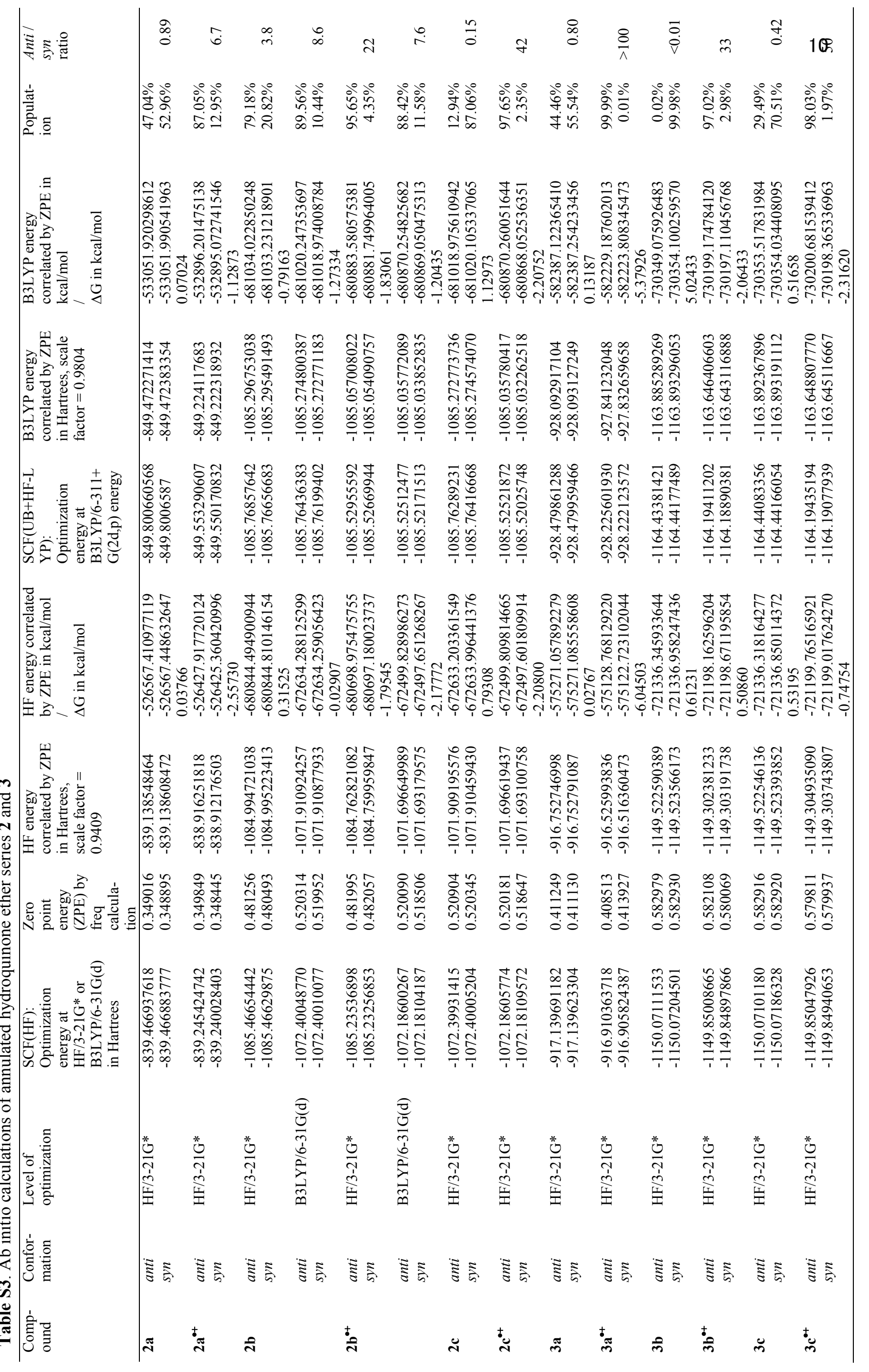




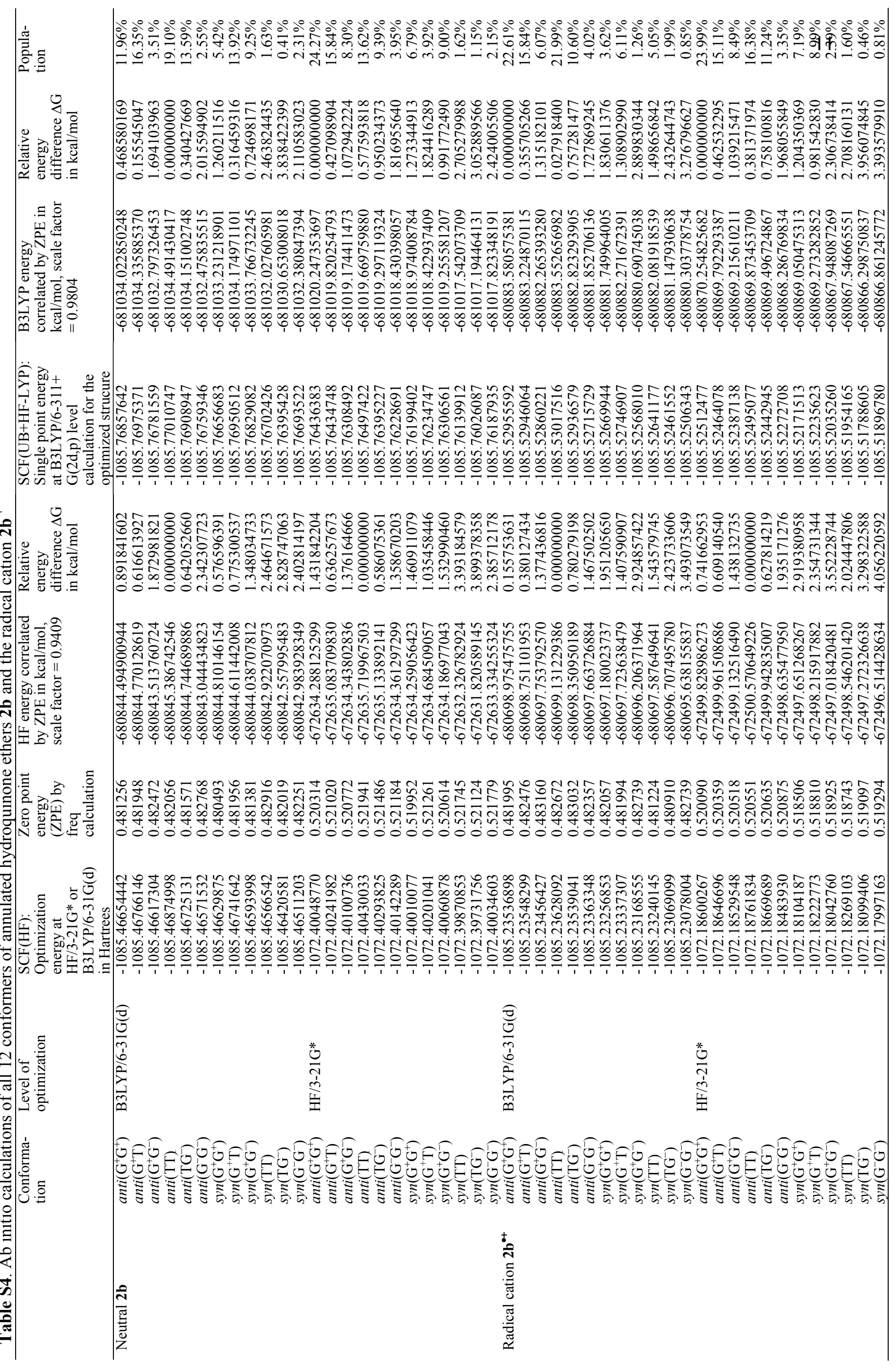




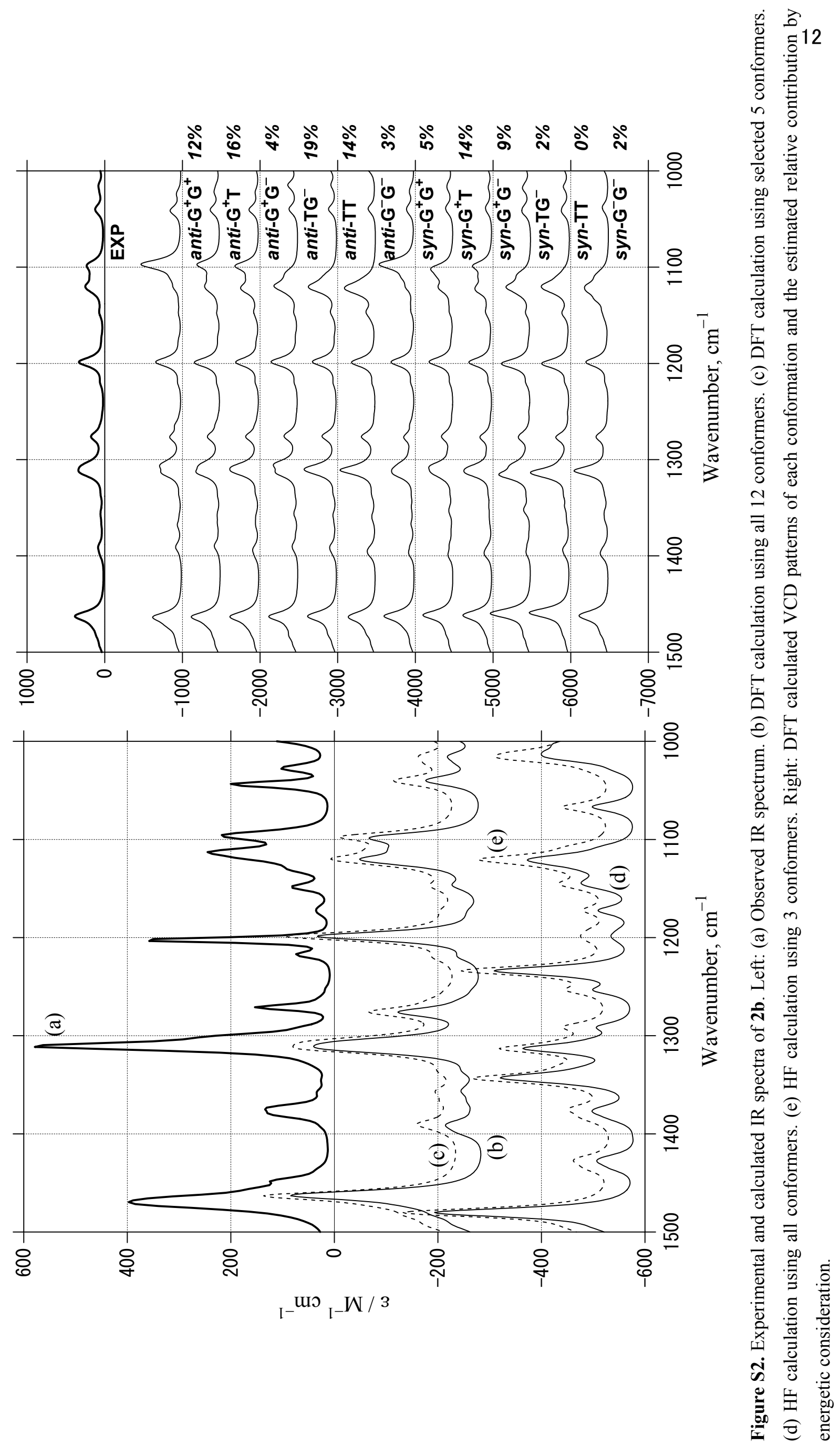




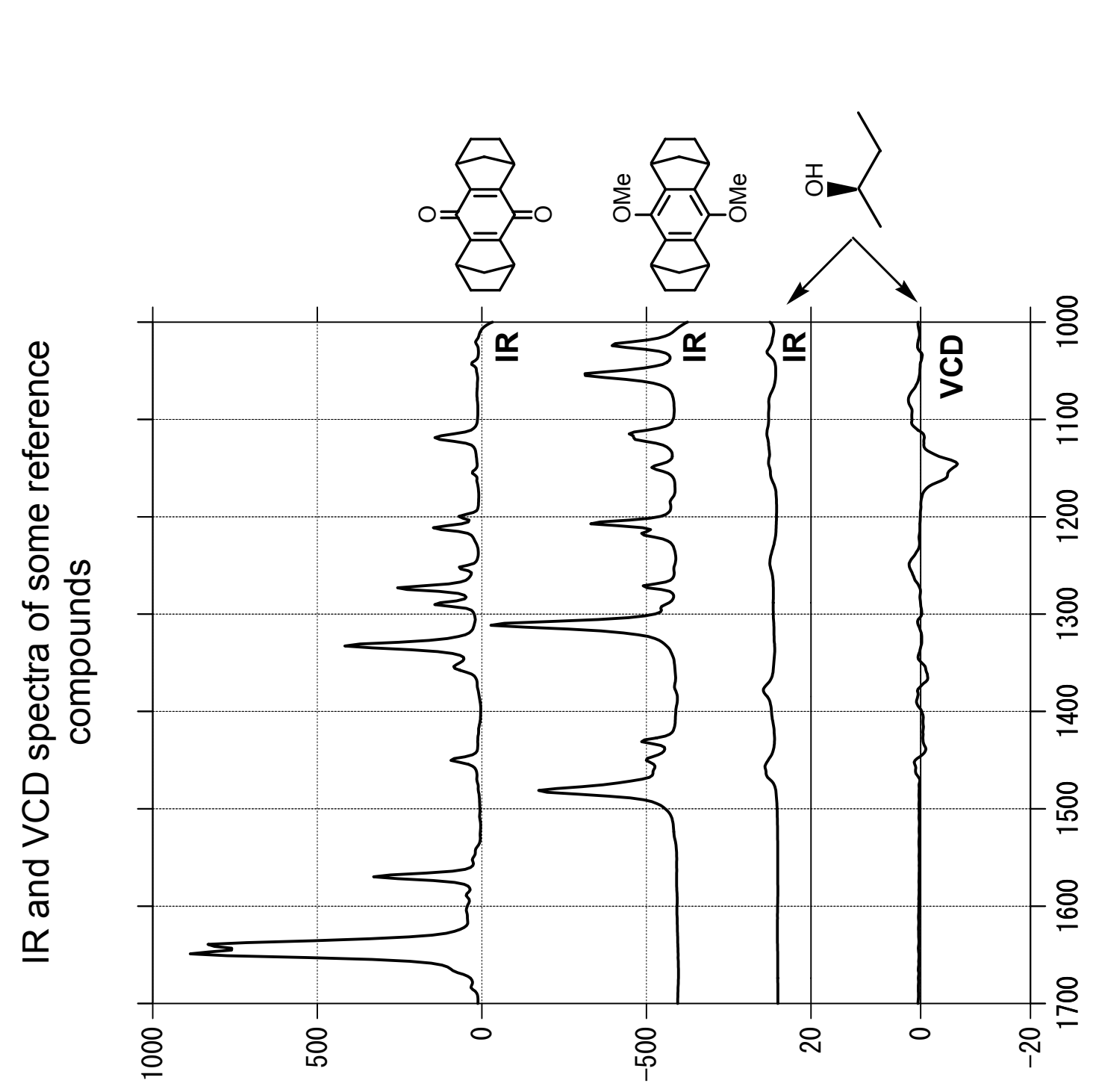



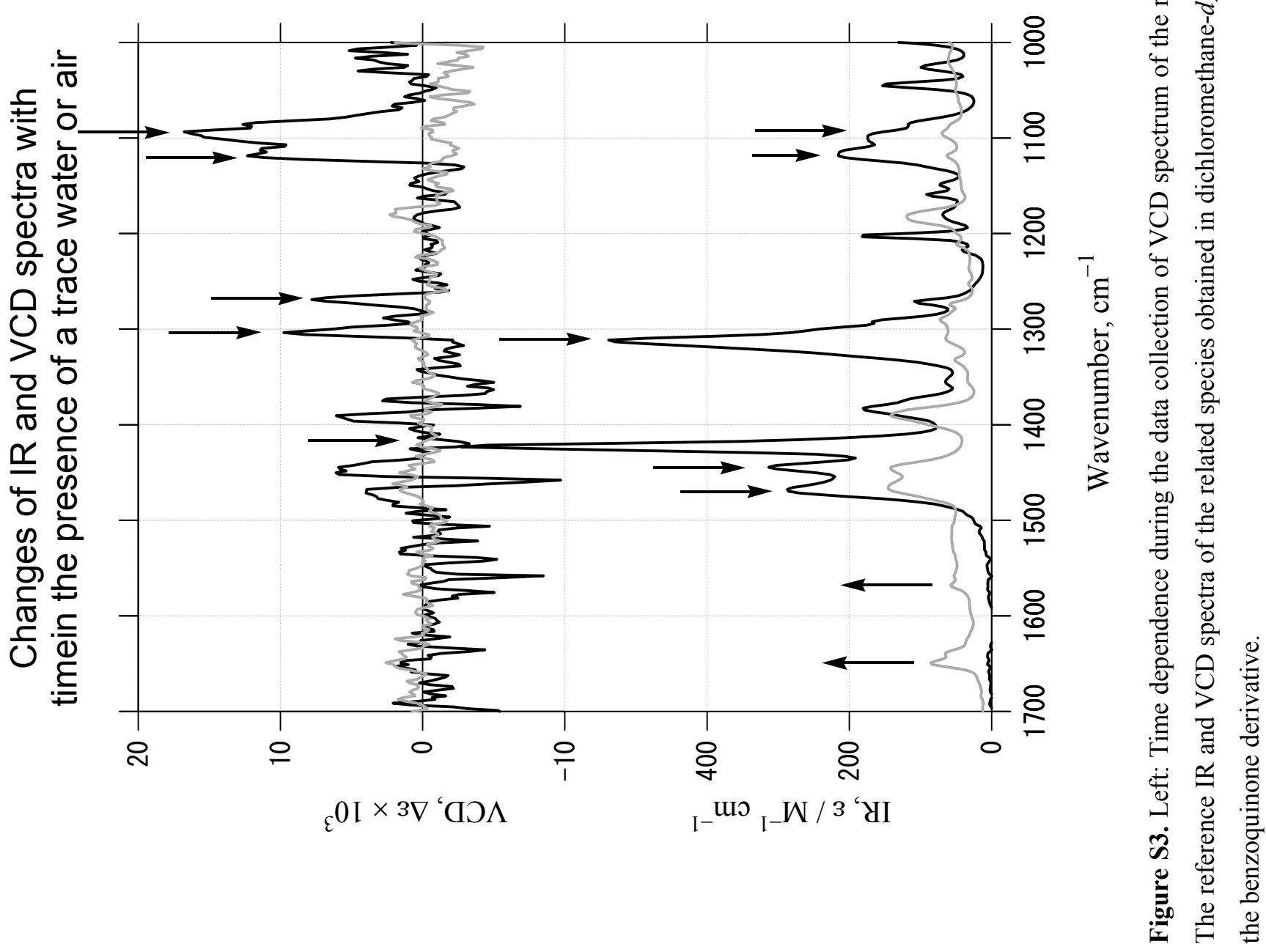

